\title{
Diagnostic utility of SALL4 in primary germ cell tumors of the central nervous system: a study of 77 cases
}

Kaiyong Mei ${ }^{1, *}$, Aijun Liu ${ }^{2, *}$, Robert W Allan ${ }^{3}$, Peng Wang ${ }^{4}$, Zhaoli Lane ${ }^{5}$, Ty W Abel ${ }^{6}$, Lixin Wei ${ }^{2}$, Hong Cheng, ${ }^{7}$ Shuangping Guo ${ }^{7}$, Yan Peng ${ }^{8}$, Dinesh Rakheja ${ }^{9}$, Min Wang ${ }^{10}$, Joe $\mathrm{Ma}^{11}$, Maria M Rodriguez ${ }^{12}$, Jianping $\mathrm{Li}^{13}$ and Dengfeng $\mathrm{CaO}^{13}$

${ }^{1}$ Department of Pathology, The Second Affiliated Hospital of Guangzhou Medical College, Guangzhou, China; ${ }^{2}$ Department of Pathology, The Chinese PLA General Hospital, Beijing, China; ${ }^{3}$ Department of Pathology, University of Florida, Gainesville, FL, USA; ${ }^{4}$ Department of Pathology, Beijing Ditan Hospital, Beijing, China; ${ }^{5}$ Department of Pathology, Henry Ford Hospital, Detroit, MI, USA; ${ }^{6}$ Department of Pathology, Vanderbilt University Medical Center, Nashville, TN, USA; ${ }^{7}$ Department of Pathology, Xijing Hospital, Xian, China; ${ }^{8}$ Department of Pathology, The University of Texas-Southwestern Medical Center, Dallas, TX, USA; ${ }^{9}$ Children's Medical Center, The University of Texas-Southwestern Medical Center, Dallas, TX, USA;

${ }^{10}$ Department of Pathology and Laboratory Medicine, University of Texas Health Science Center at Houston, Houston, TX, USA; ${ }^{11}$ Department of Pathology, Florida Hospital, Orlando, FL, USA; ${ }^{12}$ Department of Pathology, University of Miami, Miami, FL, USA and ${ }^{13}$ Department of Pathology and Immunology, Washington University School of Medicine, Saint Louis, MO, USA

Primary germ cell tumors of the central nervous system (CNS) sometimes pose diagnostic difficulty. In this study we analyzed the diagnostic utility of a novel marker, SALL4, in $\mathbf{7 7}$ such tumors (59 pure and 18 mixed) consisting of the following tumors/tumor components: 49 germinomas, 7 embryonal carcinomas, 27 yolk sac tumors, 3 choriocarcinomas, and 14 teratomas. We also stained SALL4 in 99 primary non-germ cell tumors to test SALL4 specificity. We compared SALL4 with OCT4 in all germ cell tumors and compared SALL4 with $\alpha$-fetoprotein and glypican-3 in all yolk sac tumors. The staining was semiquantitatively scored as 0 (no staining), $1+(<=30 \%), 2+(31-60 \%), 3+(61-90 \%)$, and $4+(>90 \%)$. Strong SALL4 staining was observed in all 49 germinomas $(4+$ in $48,3+$ in 1$), 7$ embryonal carcinomas (all $4+$ ), and 27 yolk sac tumors $(1+$ in $1,2+$ in $2,3+$ in $7,4+$ in 17). SALL4 staining, $1+$ weak to focally strong, was observed in 2 of 3 choriocarcinomas (in mononucleated trophoblasts) and in 9 of 14 teratomas (in primitive neuroepithelium and teratomatous glands). All germinomas and embryonal carcinomas showed strong OCT4 staining $(4+$ in all except 1 germinoma with $3+$ ), whereas other germ cell tumors were negative. Out of 27 yolk sac tumors, 26 showed positive $\alpha$-fetoprotein staining ( $1+$ in $9,2+$ in $7,3+$ in 5 , and $4+$ in 5$)$. All yolk sac tumors showed positive glypican-3 staining ( $1+$ in 6 , $2+$ in $6,3+$ in 7 , and $4+$ in 8 ). The mean percentage of yolk sac tumor cells stained was $84 \%$ with SALL4, $45 \%$ with $\alpha$-fetoprotein, and $63 \%$ with glypican-3 $(P<0.01)$. No non-germ cell tumors showed SALL4 staining. Our results indicate that SALL4 is a novel sensitive diagnostic marker for primary germ cell tumors of the CNS with high specificity. SALL4 is a more sensitive marker than $\alpha$-fetoprotein and glypican-3 for yolk sac tumors. Modern Pathology (2009) 22, 1628-1636; doi:10.1038/modpathol.2009.148; published online 9 October 2009

Keywords: germ cell tumors; central nervous system; SALL4; diagnostic marker

Correspondence: Dr D Cao, Department of Pathology and Immunology, Washington University School of Medicine, Division of Anatomic and Molecular Pathology, 660 South Euclid Avenue, Campus Box 8118, Saint Louis, MO, 63110 USA.

E-mail: dcao@path.wustl.edu

${ }^{*}$ Both authors contributed equally to this project.

Received 19 July 2009; revised 19 August 2009; accepted 24 August 2009; published online 9 October 2009
Primary germ cell tumors of the central nervous system (CNS) are rare neoplasms and include germinoma, embryonal carcinoma, yolk sac tumor, choriocarcinoma, teratoma, and mixed types. ${ }^{1}$ As with many CNS specimens, the tissue obtained for diagnosis of primary germ cell tumors is often small with mechanical crushing artifact and/or frozen 
artifacts, making it difficult to assess the morphologic features of the tumor cells. The diagnosis is sometimes further complicated by the obscuring background inflammatory and/or granulomatous infiltrate. ${ }^{2}$ For these reasons, the diagnosis of germ cell tumors in the CNS can be sometimes difficult without ancillary techniques. Nonetheless, correct pathologic diagnosis of primary germ cell tumors and distinguishing them from non-germ cell tumors is critical, as the management for these two groups of tumors is different. In difficult cases, immunohistochemical markers are often needed to facilitate the diagnosis.

Earlier markers used for diagnosing primary germ cell tumors in CNS include placental-like alkaline phosphatase, C-KIT, and $\alpha$-fetoprotein. Although these are good markers, they only show moderate sensitivity and/or specificity. ${ }^{3-8}$ More recently, novel stem cell markers, OCT4 and NANOG, have been proposed as more sensitive markers, but they were expressed only in a subset of primary germ cell tumors, such as germinoma and embryonal carcinoma, whereas other types of germ cell tumors, such as yolk sac tumors, were not immunoreactive for these markers. ${ }^{3,7,9}$ Among the germ cell tumors, yolk sac tumor poses the greatest diagnostic challenges because of its multiple histological patterns. Recently, a novel stem cell marker, SALL4, has been identified as a novel gonadal germ cell tumor marker. ${ }^{10-12}$ SALL4 was strongly positive in $>90 \%$ of the tumor cells in all gonadal yolk sac tumors, seminomas/dysgerminomas, and embryonal carcinomas. The purpose of this study is to analyze the diagnostic utility of SALL4 in a large series of 77 primary germ cell tumors of the CNS (59 pure and 18 mixed tumors). We compared SALL4 with OCT4 in all these germ cell tumors. For yolk sac tumors, we compared SALL4 with $\alpha$-fetoprotein, the most common marker for yolk sac tumors, and a recently described gonadal yolk sac tumor marker glypican3 , which has not yet been systematically analyzed in primary yolk sac tumors of the CNS. To test SALL4 specificity, we stained 99 primary CNS non-germ cell tumors.

\section{Materials and methods}

\section{Case Selection}

Approval to perform this study was obtained from the institutional review boards. The surgical pathology files (between 1990 and 2008) of the authors' institutions were searched for primary germ cell tumors of the CNS. In all, 77 such cases were identified for this study, including 59 pure ones (39 germinomas, 15 yolk sac tumors, 1 embryonal carcinoma, 1 immature teratoma, 1 mature teratoma, and 2 choriocarcinomas) and 18 mixed ones (with germinoma component in 10, yolk sac tumor component in 12, embryonal carcinoma component in 6 , immature teratoma component in 8 , mature teratoma component in 4, and choriocarcinoma component in 1; Table 1).

To test SALL4 specificity in primary CNS tumors, 99 primary non-germ cell tumors were also stained with SALL4, including 11 chordomas, 11 meningiomas (4 chordoid, 4 clear cell, 1 microcystic, and 2 fibroblastic), 8 pilocytic astrocytomas, 10 astrocytomas (6 WHO grade 2 and 4 WHO grade 3), 11 glioblastoma multiformes, 9 ependymomas (6 WHO grade 2 and 3 WHO grade 3), 6 oligodendrigliomas (2 WHO grade 2 and 4 WHO grade 3), 8 pituitary adenomas, 6 central neurocytomas, 6 pinealoblastomas, and 13 diffuse large B-cell lymphomas.

Table 1 Clinicopathological information of 18 primary mixed germ cell tumors of the central nervous system

\begin{tabular}{|c|c|c|c|c|}
\hline Case no. & Gender & Age (years) & Tumor location & Germ cell tumor components \\
\hline 1 & Male & 10 & Pineal region & Germinoma, embryonal carcinoma, yolk sac tumor, mature teratoma \\
\hline 2 & Male & 18 & Pineal region & Germinoma, immature teratoma \\
\hline 3 & Male & 8 & Pineal gland & Yolk sac tumor, immature teratoma \\
\hline 4 & Male & 33 & Sellum & Embryonal carcinoma, yolk sac tumor, immature teratoma \\
\hline 5 & Male & 8 & $\begin{array}{l}\text { Cerebrum } \\
\text { (deep parietotemporal) }\end{array}$ & Yolk sac tumor (+ germinoma)* \\
\hline 6 & Male & 15 & Pineal region & Germinoma, yolk sac tumor \\
\hline 7 & Male & 11 & Third ventricle & Yolk sac tumor, mature teratoma \\
\hline 8 & Male & 15 & Sellar/suprasellar region & Germinoma, mature teratoma \\
\hline 9 & Female & 15 & Third ventricle & Germinoma, choriocarcinoma \\
\hline 10 & Female & 8 & Sellum & Yolk sac tumor, immature teratoma, germinoma, embryonal carcinoma \\
\hline 11 & Female & 24 & Sellum & Germinoma, yolk sac tumor \\
\hline 12 & Male & 25 & Third ventricle & Yolk sac tumor, immature teratoma, embryonal carcinoma \\
\hline 13 & Female & 6 & Sellum & (Yolk sac tumor)*, embryonal carcinoma, (choriocarcinoma)* \\
\hline 14 & Male & 16 & Basal ganglia & Germinoma, yolk sac tumor \\
\hline 15 & Male & 14 & Pineal region & Germinoma, immature teratoma \\
\hline 16 & Male & 14 & Pineal region & Germinoma, yolk sac tumor, immature teratoma \\
\hline 17 & Female & 1 day & Posterior fossa & Yolk sac tumor, immature teratoma \\
\hline 18 & Male & 16 & Pineal region & Embryonal carcinoma, mature teratoma \\
\hline
\end{tabular}

*The components in parentheses marked with an asterisk were present in the tumor but not on the sections for SALL4 staining. 


\section{Immunohistochemical Staining and Evaluation}

For each case, one to two formalin-fixed paraffinembedded tissue blocks were retrieved to generate 4- $\mu \mathrm{m}$ unstained slides for immunohistochemical stains in all germ cell tumors and non-germ cell tumors with an SALL4 monoclonal antibody (WH0057167-M03, dilution 1:100, Sigma-Aldrich, St Louis, MO, USA). OCT4 staining was performed for all germ cell tumors (prediluted antibody, Ventana Medical Systems, Tuscon, AZ, USA). $\alpha$-fetoprotein (prediluted antibody, Ventana Medical Systems) and glypican-3 (prediluted antibody, Biomosaics, Burlington, VT, USA) immunostains were performed for all yolk sac tumors. Appropriate positive and negative controls were included for each run of immunostains. The immunostains were performed on a Ventana Benchmark-XT automated stainer using the Ventana ultraView DAB detection kit. The antigen retrieval was performed using Ventana Tris-based buffer solution CC1, pH 8.0 at 95-100 ${ }^{\circ} \mathrm{C}$ for $60 \mathrm{~min}$. Only nuclear staining was considered positive for SALL4 and OCT4. The staining intensity of SALL4 was scored as weak or strong. The staining pattern for $\alpha$-fetoprotein and glypican-3 was cytoplasmic. The percentage of tumor cells labeled with each marker was semiquantitatively scored as 0 (no tumor cells staining), $1+(<=30 \%), 2+(31-60 \%), 3+(61-90 \%)$, and $4+$ $(>90 \%)$.

\section{Statistical Analysis}

The Fisher's exact test was used to compare the staining results of SALL4 with OCT4, $\alpha$-fetoprotein, or glypican-3. Paired $t$-test was used to compare the mean percentage of tumor cells stained with SALL4 with $\alpha$-fetoprotein or glypican-3. A $P$-value of $<0.05$ was considered statistically significant.

\section{Results}

\section{SALL4 in Germinomas}

There were 49 germinomas (39 pure tumors and 10 as a component in mixed germ cell tumors) in this study. Of these 39 patients with pure germinomas, 33 were males (mean age 20 years old, range 8-48 years) and 6 were females (mean age 17 years, range 5-46). These pure germinomas were located in the pineal region ( $n=27,26$ males and 1 female), sellar/ suprasellar region ( $n=7,2$ males and 5 females), thalamus and hypothalamus $(n=3$, all males $)$, tectum $(n=1$, male), and third ventricle $(n=1$, male). Out of 10 mixed germ cell tumors containing seminoma, 8 occurred in males (mean age 17 years, range 10-18) and the remaining 2 occurred in females (15- and 24-year old, respectively; Table 1).
Of these 49 germinomas, 48 (98\%) showed $4+$ strong SALL4 staining (Figure 1) and 1 showed $3+$ strong SALL4 staining (80\% tumor cells). The staining pattern was nuclear with a clean background. The background lymphocytes, fibroblasts, endothelial cells, glial tissue, and so on were negative for SALL4 staining.

\section{SALL4 in Embryonal Carcinomas}

In all, seven embryonal carcinomas were included for this study including 1 pure tumor (in the sellar region of a 10 year-old female) and 6 as a component in mixed germ cell tumors (Table 1). All seven embryonal carcinomas showed $4+$ strong SALL4 staining (Figure 1).

\section{SALL4 in Yolk Sac Tumors}

We included 27 yolk sac tumors in this study including 15 pure ones and 12 as a component in mixed germ cell tumors. The mean age for 15 patients (11 males and 4 females) with pure yolk sac tumors was 16 years (age range 11 months to 29 years). The tumor locations of these pure yolk sac tumors included pineal region $(n=6)$, sellar/suprasellar region $(n=2)$, ventricle $(n=3)$, skull base $(n=2)$, pellucidum $(n=1)$, and cerebellum $(n=1)$. The 12 patients (10 males and 2 females) with mixed germ cell tumors containing yolk sac tumor component had a mean age of 14.3 years (range 1 day to 33 years; Table 1).

Of these 27 yolk sac tumors, strong positive SALL4 staining was observed in all cases, including $1+$ in $1(4 \%), 2+$ in $2(7 \%), 3+$ in $7(26 \%)$, and $4+$ in $17(63 \%)$ cases (Figure 2).

\section{SALL4 in Teratomas}

In all, 14 teratomas were included in this study consisting of 2 pure ones and 12 as a component in mixed germ cell tumors. The two pure teratomas include one mature (in the pineal region of a 3 -month-old girl) and one immature one (in the pineal region of a 3-year-old boy). The 12 teratomatous components in mixed germ cell tumors were mature in four cases and were immature in eight cases. The age and gender information of these patients with mixed germ cell tumors containing a teratomatous component is listed in Table 1.

All five mature teratomas/teratomatous components were negative for SALL4 staining. In all, seven of the $9(78 \%)$ immature teratomas/teratomatous components showed $1+$ SALL4 staining: weak to focally strong SALL4 staining in the immature primitive neuroepithelium and blastema-like area in four cases (5-10\% cells; Figure 3), weak staining in low-grade immature mesenchyme in 2 (2 and $5 \%$ 


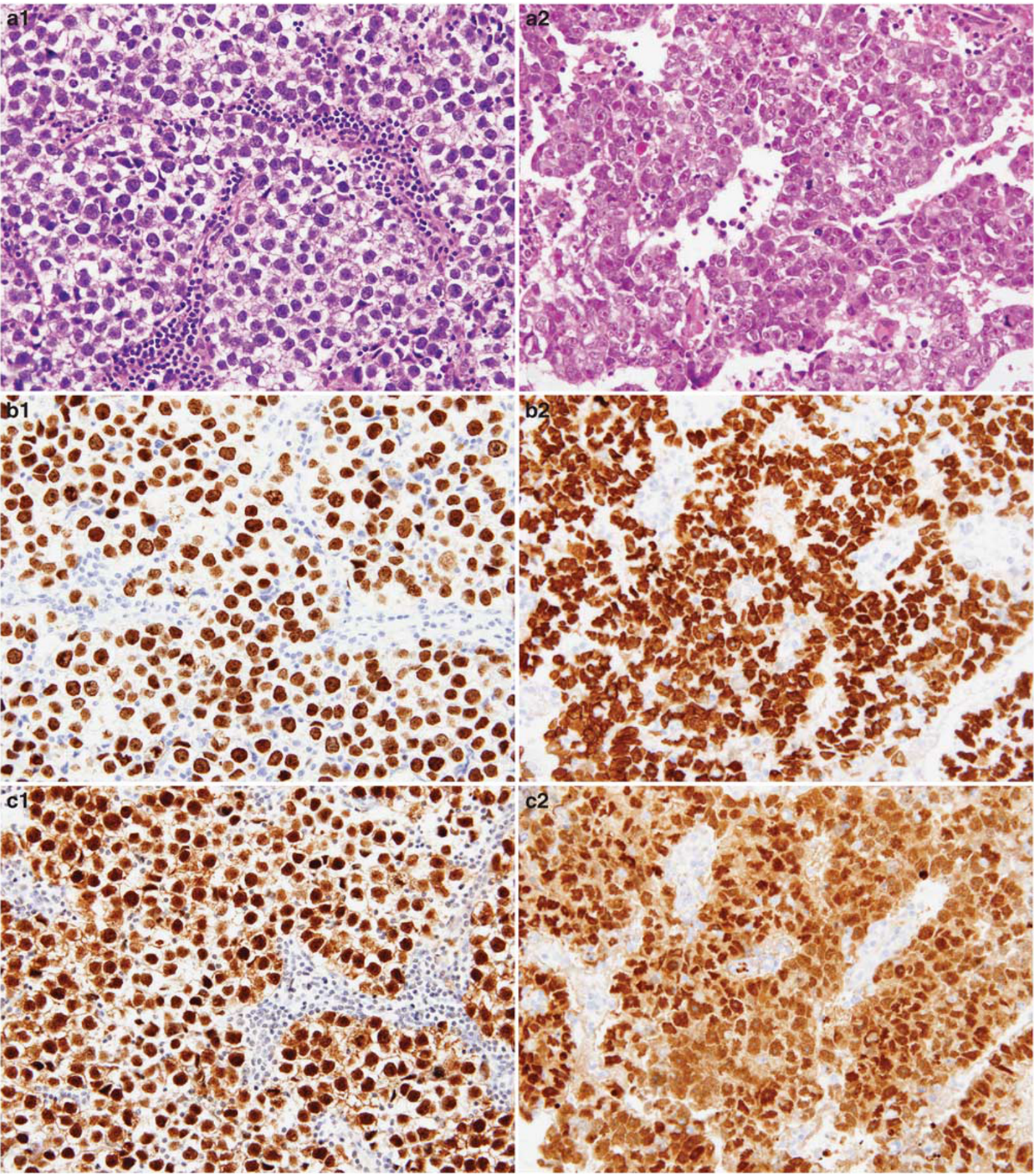

Figure 1 Immunohistochemical staining of SALL4 in germinoma (a1, H\&E) and embryonal carcinoma (a2, H\&E). Strong SALL4 staining was observed in all germinomas (b1) and embryonal carcinomas (b2). The tumor cells were confirmed by their strong staining with OCT4 $(\mathbf{c 1}, \mathbf{c 2})$.

cells, respectively), and in teratomatous glands in 2 $(5 \%$ gland cells each; Figure 3$)$ cases. Other teratomatous components, including squamous epithelium and nests, cartilage, smooth muscle, fibroadipose tissue, and mature neural tissue, were all negative for SALL4.

\section{SALL4 in Choriocarcinomas and Syncytiotrophoblasts}

Two pure choriocarcinomas (both in the pineal region in males, 12 years and 23 years old, respectively) and one choriocarcinomatous component in a mixed germ cell tumor (in the third 


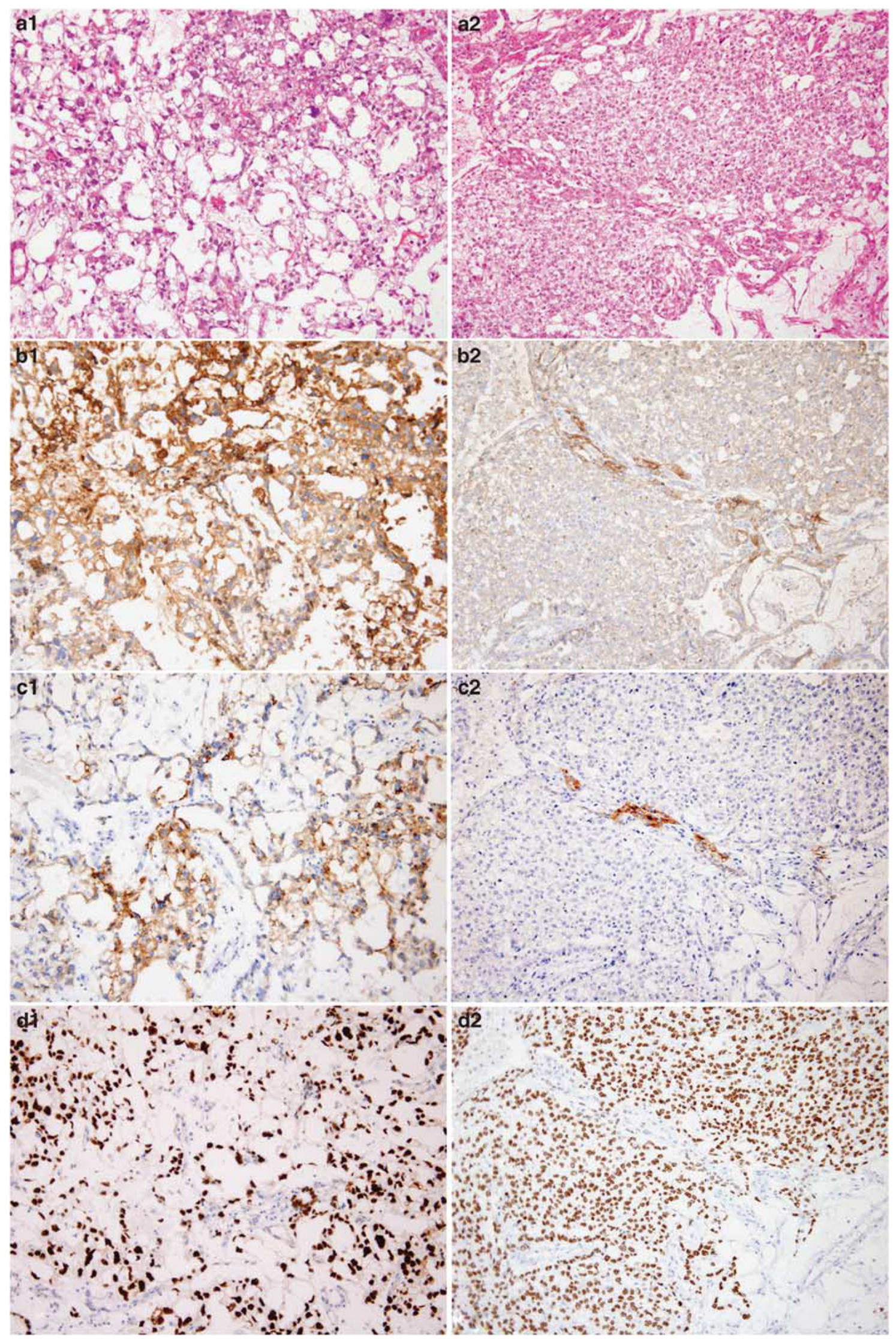




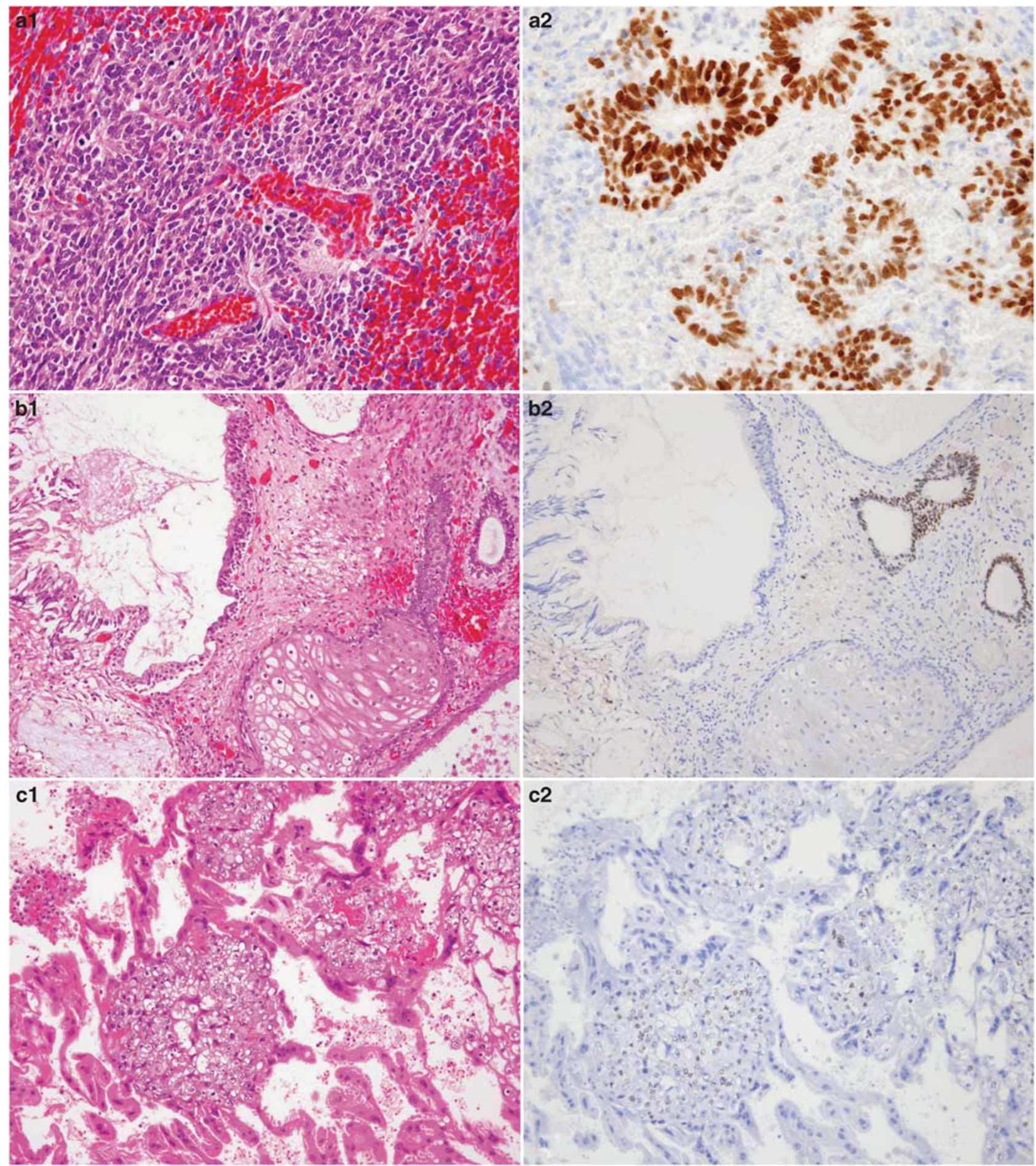

Figure 3 Immunohistochemical staining of SALL4 in primary teratomas (a1, immature neuroepithelium and b1, mature elements in an immature teratoma) and choriocarcinomas (c1) of the central nervous system. Most immature teratomas showed focal weak-to-strong SALL4 staining, typically in the primitive neuroepithelium (A2), but the teratomatous glands in an immature teratoma may also show focal weak SALL4 staining (b2). Focal weak SALL4 staining was observed in the mononucleated trophoblasts in two-thirds of the choriocarcinomas, but syncytiotrophoblasts were negative (c2).

Figure 2 Yolk sac tumor can show multiple histological patterns, such as microcystic (a1, H\&E) and solid and microcystic pattern (a2, H\&E). Although few yolk sac tumors showed diffuse staining for $\alpha$-fetoprotein (b1), most of these tumors typically showed focal staining (b2). Positive glypican-3 staining (c1, c2) was observed in all yolk sac tumors but only one-third of them showed diffuse staining (c1), whereas in the remaining cases the staining was typically focal (c2). In contrast, strong positive SALL4 staining was observed in $>90 \%$ of the tumor cells in two-thirds (17 of 27) of the yolk sac tumors (d1, d2). 
Table 2 Comparison of SALL4 with OCT4 in primary germ cell tumors of the central nervous system

\begin{tabular}{|c|c|c|c|}
\hline Germ cell tumor or component & $S A L L 4$ & OCT4 & $\mathrm{P}$-value \\
\hline Germinoma $(n=49)$ & $49 / 49(100 \%)$ & $49 / 49(100 \%)$ & 1.00 \\
\hline Embryonal carcinoma $(n=7)$ & $7 / 7(100 \%)$ & $7 / 7(100 \%)$ & 1.00 \\
\hline Yolk sac tumor $(n=27)$ & $27 / 27(100 \%)$ & $0 / 27$ & $<0.001$ \\
\hline Teratoma $(n=14)$ & $9 / 14(64 \%)^{a}$ & $0 / 14$ & $<0.01$ \\
\hline Choriocarcinoma $(n=3)$ & $2 / 3(67 \%)^{a}$ & $0 / 3$ & 0.4 \\
\hline
\end{tabular}

${ }^{\mathrm{a}}$ The staining was focal, typically weak.

Table 3 Comparison of SALL4 with $\alpha$-fetoprotein and glypican-3 in 27 primary yolk sac tumors of the central nervous system

\begin{tabular}{|c|c|c|c|c|c|c|}
\hline \multirow[t]{2}{*}{ Marker } & \multicolumn{5}{|c|}{ Staining pattern* } & \multirow[t]{2}{*}{$\mathrm{P}$-value $S A L L 4$ vs } \\
\hline & 0 & $1+$ & $2+$ & $3+$ & $4+$ & \\
\hline SALL4 & 0 & $1(4 \%)$ & $2(7 \%)$ & $7(26 \%)$ & $17(63 \%)$ & \\
\hline$\alpha$-fetoprotein & $1(4 \%)$ & $9(33 \%)$ & $7(26 \%)$ & $5(19 \%)$ & $5(19 \%)$ & $<0.01$ \\
\hline Glypican-3 & 0 & $6(22 \%)$ & $6(22 \%)$ & $7(26 \%)$ & $8(30 \%)$ & $<0.01$ \\
\hline
\end{tabular}

*The staining pattern is as follows: 0 : no tumor cell staining; $1+: \leqslant 30 \%$ tumor cells staining; $2+: 31-60 \%$ tumor cells staining; $3+: 61-90 \%$ tumor cells staining; $4+:>90 \%$ tumor cells staining.

ventricle in association with germinoma in a 15year-old female; Table 1) were included in this study. One of the two pure choriocarcinomas showed $1+$ weak SALL4 staining in the mononucleated trophoblasts (20-25\% cells; Figure 3). In the case of choriocarcinoma associated with a mixed GCT, the choriocarcinoma component showed $1+(20 \%$ tumor cells $)$ weak staining in the mononucleated trophoblasts. Syncytiotrophoblasts were negative for SALL4 staining in all three cases.

\section{Comparison of SALL4 with OCT4 in Primary Germ Cell Tumors of the CNS}

We performed OCT4 immunohistochemical staining for all germ cell tumors. OCT4 was strongly positive in all 49 germinomas $(4+$ in 48 and $3+$ in 1$)$ and 7 embryonal carcinomas $(4+$ in all 7; Figure 1$)$. No OCT4 staining was observed in any yolk sac tumor, teratoma, or choriocarcinoma. Table 2 summarizes the comparison of SALL4 result with OCT4 in all germ cell tumors.

\section{Comparison of SALL4 to $\alpha$-Fetoprotein and Glypican-3 in Yolk Sac Tumors}

Out of 27 yolk sac tumors, 26 (96\%) were positive for $\alpha$-fetoprotein, including $1+$ in $9(33 \%)$ cases, 2 + in $7(26 \%), 3+$ in $5(19 \%)$, and $4+$ in $5(19 \%$; Table 3 and Figure 2). One yolk sac tumor was negative for $\alpha$-fetoprotein staining. In a few cases, there was some background $\alpha$-fetoprotein staining. All yolk sac tumors showed positive glypican-3 staining (Figure 2), including $1+$ in $6(22 \%), 2+$ in $6(22 \%), 3+$ in $7(26 \%)$, and $4+$ in $8(30 \%)$ tumors.
As described above, strong SALL4 staining was observed in all 27 yolk sac tumors $(100 \%$ sensitivity). Compared with $\alpha$-fetoprotein and glypican-3, more yolk sac tumors showed $3+(7 / 27$ or $26 \%)$ and $4+(17 / 27$ or $63 \%)$ SALL4 staining (Table 3, $P<0.01)$. The mean percentage of tumor cells showing SALL4 staining was $84 \%$ (range $5-100 \%$ ), whereas it was $45 \%$ (range $0-98 \%$ ) for $\alpha$-fetoprotein and $63 \%$ (range $2-95 \%, 2$ with less than $10 \%$ tumor cells stained) for glypican-3 $(P<0.01$ between SALL4 and $\alpha$-fetoprotein or glypican-3).

\section{SALL4 in Primary Non-Germ Cell Tumors of the Central Nervous System}

All 99 primary non-germ cell tumors were negative for SALL4 staining.

\section{Discussion}

Primary germ cell tumors of the CNS are rare neoplasms and may pose diagnostic challenges because of the limited tissue, and crushing and frozen section artifacts. In difficult cases, ancillary techniques, such as immunohistochemistry, are often needed to facilitate the diagnosis. In this study we analyzed the diagnostic utility of a novel stem cell marker, SALL4, in a large series of 77 primary germ cell tumors of the CNS. Our results have shown that strong SALL4 staining was observed in all 49 germinomas $(3+$ in 1 and $4+$ in 48), 7 embryonal carcinomas $(4+$ in all), and 27 yolk sac tumors $(1+$ in $1,2+$ in $2,3+$ in 7 , and $4+$ in 17$)$. Focal SALL4 staining $(<30 \%$ tumor cells) was also observed in 2 of 3 choriocarcinomas and 9 of 14 
teratomas. These findings indicate that SALL4 is a novel diagnostic marker for primary germ cell tumors of the CNS, with $100 \%$ sensitivity for germinomas, embryonal carcinomas, and yolk sac tumors of the CNS. This utility can be used to confirm a primary CNS tumor to be a germ cell tumor. A primary tumor of the CNS that is negative for SALL4 is highly unlikely to be germinoma, embryonal carcinoma, or yolk sac tumor.

Of the primary germ cell tumors of the CNS, yolk sac tumor probably poses the greatest diagnostic challenges because of its multiple histological patterns. Primary yolk sac tumors of the CNS are also very rare. ${ }^{1}$ In our study including 13 major medical centers, we only collected 27 in-house cases (15 pure and 12 as component in mixed germ cell tumors). In spite of these, presence of a yolk sac tumor component in a primary germ cell tumor of the CNS often indicates a more malignant behavior than germinoma and teratoma. ${ }^{13,14}$ Therefore, accurate diagnosis of yolk sac tumor or component is important. Morphologically, besides mimicking other types of germ cell tumors, yolk sac tumor may also mimic non-germ cell tumors, such as chordoma and chordoid meningioma. Traditionally, $\alpha$-fetoprotein has been often used to diagnose intracranial yolk sac tumor, but the staining was typically focal and patchy as observed by us and others, ${ }^{4,7,8,15}$ calling for more sensitive markers. In addition, we observed that $\alpha$-fetoprotein staining was sometimes associated with background staining, similar to that observed in gonadal yolk sac tumors, ${ }^{10,11,16,17}$ making it difficult to interpret. Recently, glypican-3 has been described as a novel marker for testicular yolk sac tumors, ${ }^{18}$ but its experience in primary yolk sac tumors of the CNS is very limited, with only two cases studied. ${ }^{19}$ In our study, all 27 yolk sac tumors were positive for glypican-3; however, 12 of 27 (44\%) yolk sac tumors showed staining in $<60 \%$ of the tumor cells. In contrast, only 3 of $27(11 \%)$ yolk sac tumors showed SALL4 staining in $<60 \%$ of the tumor cells and 17 of $27(63 \%)$ yolk sac tumors showed SALL4 staining in $>90 \%$ of the tumor cells. Quantitatively, the approximate mean percentage of tumor cells stained with SALL4 was also much higher $(84 \%)$ than with $\alpha$-fetoprotein (45\%) and glypican-3 $(63 \% ; P<0.001)$. Therefore, SALL4 is a more sensitive marker than $\alpha$-fetoprotein and glypican-3 for primary yolk sac tumors of the CNS.

SALL4 is a zinc-finger transcription factor that shares homology with the Drosophila spalt (sal) gene, and is essential for development. ${ }^{20-23}$ SALL4 forms a regulatory circuit with OCT4, NANOG, and SOX2 to maintain embryonic stem cell pluripotency and self-renewal. ${ }^{24-29}$ In this self-stabilizing network, SALL4 regulates OCT4 transcription, suggesting acting upstream of OCT $4 .{ }^{28}$ In this study we have shown that SALL4 has a broader expression pattern than OCT4 in primary germ cell tumors of the CNS. OCT4 only labeled germinomas and embryonal carcinomas, whereas SALL4 not only stained germinomas and embryonal carcinomas but also all yolk sac tumors and some teratomas (9/14) and choriocarcinomas (2/3). It is unclear whether SALL4 regulates OCT4 in germ cell tumors of the CNS, as it does in embryonic stem cells. The difference between SALL4 and OCT4 in germ cell tumors can be used to help subtype primary germ cell tumors of the CNS. For example, solid yolk sac tumor may mimic solid embryonal carcinoma and solid germinoma. An immunohistochemical panel, including SALL4, OCT4, and CD30, will help to solve this diagnostic difficulty. Germinoma will be positive for both SALL4 and OCT4 but negative for CD30, whereas embryonal carcinoma will show SALL4+ /OCT4 + /CD30 + profile and yolk sac tumor will show SALL4 + /OCT4-/CD30- profile.

Previous studies have shown that SALL4 is a highly sensitive marker for gonadal seminoma/ dysgerminoma, embryonal carcinomas, and yolk sac tumor as well as their metastatic form, including those metastasizing to the CNS. ${ }^{10-12}$ In the current study we have shown $100 \%$ sensitivity of SALL4 for their counterparts in the CNS. Such high sensitivity was also observed in primary mediastinal seminomas, embryonal carcinomas, and yolk sac tumors (Cao D, unpublished results). Therefore, the high ( $100 \%$ so far) sensitivity of SALL4 for seminoma/ dysgerminoma/germinoma, embryonal carcinoma, and yolk sac tumor, is not only observed in the gonads but also in extragonadal sites. In previous studies, of the 461 non-germ cell tumors (273 carcinomas of almost all major types, 92 sarcomas of almost all types, 12 melanomas and 13 mesotheliomas, and 71 gonadal non-carcinomatous nongerm cell tumors), only 13 carcinomas and 1 sarcoma showed focal SALL4 staining in $<30 \%$ of the tumor cells. ${ }^{10-12}$ In this study none of the 99 nongerm cell tumors of all major types of the CNS showed positive SALL4 staining. Although further studies to include more non-germ cell tumors, especially those rare types, are needed to further delineate SALL4 specificity in tumor pathology, these results do indicate that besides its high sensitivity, SALL4 is also relatively specific for both gonadal and extragonadal germ cell tumors. In the literature, the only other types of non-germ cell tumors that were immunohistochemically positive for SALL4 staining were precursor B-lymphoblastic lymphoma and acute myeloid leukemia, whereas other types of hematolymphoid tumors were negative. ${ }^{30}$ Distinction of these tumors from germ cell tumors can usually be made on clinical presentation, morphologic findings, and, in difficult cases, immunohistochemistry for pan B cell or myeloid leukemia markers.

In summary, we analyzed SALL4 expression in a large series of 77 primary germ cell tumors and 99 non-germ cell tumors of the CNS. Strong SALL4 staining was observed not only in all germinomas and embryonal carcinomas, but also in all yolk sac 
tumors of the CNS. In addition, focal SALL4 staining was also observed in some teratomas and choriocarcinomas. In contrast, none of these 99 primary non-germ cell tumors was positive for SALL4. Our results indicate that SALL4 is a novel, sensitive diagnostic marker for primary germ cell tumors of the CNS with relatively high specificity. SALL4 is more sensitive than $\alpha$-fetoprotein and glypican-3 for primary yolk sac tumor of the CNS.

\section{Disclosure/conflict of interest}

The authors declare no conflict of interest.

\section{References}

1 Louis DN, Ohgaki H, Wiestler O, et al. WHO Classification of Tumors of the Central Nervous System, WHO Publication Center: Albany, NY, 2007;197-204.

2 Kraichoke S, Cosgrove M, Chandrasoma PT. Granulomatous inflammation in pineal germinoma. A cause of diagnostic failure at stereotaxic brain biopsy. Am J Surg Pathol 1988;12:655-660.

3 Hattab EM, Tu PH, Wilson JD, et al. OCT4 immunohistochemistry is superior to placental alkaline phosphatase (PLAP) in the diagnosis of central nervous system germinoma. Am J Surg Pathol 2005;29:368-371.

4 Ho DM, Liu HC. Primary intracranial germ cell tumor. Pathologic study of 51 patients. Cancer 1992;70: 1577-1584.

5 Iczkowski KA, Butler SL, Shanks JH, et al. Trials of new germ cell immunohistochemical stains in 93 extragonadal and metastatic germ cell tumors. Hum Pathol 2008;39:275-281.

6 Nakamura H, Takeshima H, Makino K, et al. C-kit expression in germinoma: an immunohistochemistrybased study. J Neurooncol 2005;75:163-167.

7 Ngan KW, Jung SM, Lee LY, et al. Immunohistochemical expression of OCT4 in primary central nervous system germ cell tumours. J Clin Neurosci 2008;15: 149-152.

8 Shinoda J, Miwa Y, Sakai N, et al. Immunohistochemical study of placental alkaline phosphatase in primary intracranial germ-cell tumors. J Neurosurg 1985;63: 733-739.

9 Santagata S, Hornick JL, Ligon KL. Comparative analysis of germ cell transcription factors in CNS germinoma reveals diagnostic utility of NANOG. Am J Surg Pathol 2006;30:1613-1618.

10 Cao D, Guo S, Allan RW, et al. SALL4 is a novel sensitive and specific marker of ovarian primitive germ cell tumors and is particularly useful in distinguishing yolk sac tumor from clear cell carcinoma. Am J Surg Pathol 2009;33:894-904.

11 Cao D, Li J, Guo CC, et al. SALL4 is a novel diagnostic marker for testicular germ cell tumors. Am J Surg Pathol 2009;33:1065-1077.

12 Cao D, Humphrey PA, Allan RW. SALL4 is a novel sensitive and specific marker for metastatic germ cell tumors, with particular utility in detection of metastatic yolk sac tumors. Cancer 2009;115:2640-2651.

13 Matsutani M, Sano K, Takakura K, et al. Primary intracranial germ cell tumors: a clinical analysis of 153 histologically verified cases. Neurosurg J 1997;86: $446-455$.

14 Sawamura Y, Ikeda J, Shirato H, et al. Germ cell tumours of the central nervous system: treatment consideration based on 111 cases and their long-term clinical outcomes. Eur J Cancer 1998;34:104-110.

15 Bjorsson J, Bernard WS, Okazaki H, et al. Intracranial germ cell tumors: pathobiological and immunohistochemical aspects of 70 cases. J Neuropathol Exp Neurol 1985;44:32-46.

16 Esheba GE, Pate LL, Longacre TA. Oncofetal protein glypican-3 distinguishes yolk sac tumor from clear cell carcinoma of the ovary. Am J Surg Pathol 2008;32: 600-607.

17 Ramalingam P, Malpica A, Silva EG, et al. The use of cytokeratin 7 and EMA in differentiating ovarian yolk sac tumors from endometrioid and clear cell carcinomas. Am J Surg Pathol 2004;28: 1499-1505.

18 Zynger DL, Dimov ND, Luan C, et al. Glypican 3: a novel marker in testicular germ cell tumors. Am J Surg Pathol 2006;30:1570-1575.

19 Zynger DL, Everton MJ, Dimov ND, et al. Expression of glypican 3 in ovarian and extragonadal germ cell tumors. Am J Clin Pathol 2008;130:224-230.

20 Jürgens G. Head and tail development of the Drosophila embryo involves spalt, a novel homeotic gene. EMBO J 1988;7:189-196.

21 Kohlhase J, Heinrich M, Schubert L, et al. Okihiro syndrome is caused by SALL4 mutations. Hum Mol Genet 2002;11:2979-2987.

22 Reuter D, Schuh R, Jäckle H. The homeotic gene spalt (sal) evolved during Drosophila speciation. Proc Natl Acad Sci USA 1989;86:5483-5486.

23 Sakaki-Yumoto M, Kobayashi C, Sato A, et al. The murine homolog of SALL4, a causative gene in Okihiro syndrome, is essential for embryonic stem cell proliferation, and cooperates with Sall1 in anorectal, heart, brain and kidney development. Development 2006;133:3005-3013.

24 Wang J, Rao S, Chu J, et al. A protein interaction network for pluripotency of embryonic stem cells. Nature 2006;444:364-368.

25 Warren M, Wang W, Spiden S, et al. A Sall4 mutant mouse model useful for studying the role of Sall4 in early embryonic development and organogenesis. Genesis 2007;45:51-58.

26 Wong CC, Gaspar-Maia A, Ramalho-Santos M, et al. High-efficiency stem cell fusion-mediated assay reveals Sall4 as an enhancer of reprogramming. PLoS ONE 2008;3:e1955.

$27 \mathrm{Wu} \mathrm{Q}$, Chen X, Zhang J, et al. Sall4 interacts with Nanog and co-occupies Nanog genomic sites in embryonic stem cells. J Biol Chem 2006;281: 24090-24094.

28 Zhang J, Tam WL, et al. Sall4 modulates embryonic stem cell pluripotency and early embryonic development by the transcriptional regulation of Pou5f1. Nat Cell Biol 2006;8:1114-1123.

29 Zhou Q, Chipperfield H, Melton DA, et al. A gene regulatory network in mouse embryonic stem cells. Proc Natl Acad Sci USA 2007;104:16438-16443.

30 Cui W, Kong NR, Ma Y, et al. Differential expression of the novel oncogene, SALL4, in lymphoma, plasma cell myeloma, and acute lymphoblastic leukemia. Mod Pathol 2006;19:1585-1592. 